\title{
Study on the Simulation of the APT Rough Tracing System Based on the Predictive Control Technology
}

\author{
Mingqiu Li \\ College of Electronic Information Engineering, Changchun University of Science and Technology \\ Changchun 130022, China
}

Received: December 14, $2011 \quad$ Accepted: December 24, $2011 \quad$ Published: March 1, 2012

doi:10.5539/cis.v5n2p22 URL: http://dx.doi.org/10.5539/cis.v5n2p22

\begin{abstract}
Laser space offers an attractive alternative in the military fields for its many advantages of wide bandwidth, high code rate, low power consumption and large capacity. Because the narrow laser beam will lead to many challenges in laser beam pointing, therefore, APT (acquisition, pointing and tracking) technology is one of key technology for the laser space communication. The ATP rough tracing servo turntable studied in this paper adopts two-axes-four-frame structure. In order to increase the tracking precision, the predictive control algorithm is adopted in the rough tracking position loop. The simulation result shows that, comparing with common PI controller, the predictive control algorithm could improve the dynamic response characteristics of the system and increase the tracking precision.
\end{abstract}

Keywords: Laser space communication, Rough tracing, Predictive control technology

\section{Introduction}

APT system generally needs higher acquiring scope and control accuracy. Therefore, it usually adopts compound axis structure. The rough tracing system should have large dynamic scope, narrow control bandwidth and low resonance frequency. It mainly carries out the optic axis's initial pointing and realizes acquiring and rough tracing. The position loop of rough tracing system usually adopts PI control algorithm or lag-leading control algorithm. The fractional order control algorithm was also used in the APT rough tracing system and achieved good control effect. In this paper, the dynamic matrix control (DMC) algorithm will be used into rough tracing system to improve the dynamic response characteristics of the rough tracing system (Hu, 2005; Toyoda Masahiro, 2006; Liu, 2006; Shinhak Lee).

\section{Components of the APT System and Control Requirements}

The components of the compound axis APT system are shown in Figure 1, and the rough tracking system is composed by the gimbals servo turntable and the rough tracking CCD. The main components of the fine tracing system are the fast steering mirror and the fine tracing detector, and the fine tracing system could further correct the residual error which could not be compensated by the coarse tracing loop, to satisfy the aiming and tracing accuracy required by the system.

The sample period of the rough tracking system is $0.02 \mathrm{~S}$. The overshoot should be lower than $20 \%$. The cut-off frequency should be lower than $5.8 \mathrm{~Hz}$ according to the relation of sample period, cut-off frequency and overshoot. The bandwidth of the rough tracking system should be lower than $10 \mathrm{~Hz}$. In order to reduce the time delay of motor, broaden the speed regulation scope, restrain the influence of moment of force fluctuation and avoid the resonance of position loop and speed loop, the bandwidth of speed loop should be more than 3 times the bandwidth of speed loop of position loop (Tzung-Hsien Ho, 2005; Tong Luo, 2002; Shinhak Lee, 2000).

\section{Design of Speed Loop of Rough Tracking System}

From Figure 1, the rough tracking system is composed by the speed loop and the position loop.

The mathematical model of the speed loop is

$$
W_{m}(s)=\frac{1 / K_{b}}{\left(T_{m} s+1\right)\left(T_{e} s+1\right)}=\frac{0.1483}{(1+3 s)(1+0.0031 s)}
$$


To make the compensated speed loop satisfy the bandwidth requirement, the cut-off frequency of the compensated speed loop is $\omega_{c}=100 \mathrm{rad} / \mathrm{s}$, and the compensation system of the speed loop designed by adopting the frequency characteristic method of the classical control theory is

$$
G_{c}(s)=\frac{4046(0.01 s+1)(0.0031 s+1)}{(0.02 s+1)(0.002 s+1)}
$$

The step response of the compensated speed loop is shown in Figure 2. The open loop and closed loop frequency characteristic of compensated speed loop is shown in Figure 3 and Figure 4. From which we can see that the overshoot of speed loop is $17 \%$, the settling time is $0.09 \mathrm{~S}$, the phase margin is $59.7^{\circ}$, the bandwidth is $168 \mathrm{rad} / \mathrm{s} \approx 26.8 \mathrm{~Hz}$. It can satisfy the requirements of the rough tracking system.

According to the error index of the tracking system and the maximum work angle speed and the maximum acceleration of the system, the open loop gain required by the system can be confirmed by $\mathrm{K}=2000$.

The uncompensated transfer function of the position loop is

$$
P_{0}(s)=\frac{2000(6 s+600)}{s\left(0.0001 s^{3}+0.066 s^{2}+8.02 s+601\right)}
$$

\section{Design of Position Loop of Rough Tracking System Based on Predictive Control Technology}

In the designing procedure of predictive control algorithm, the predictive model is established according to the features of controlled process. The future output of system can be predicted by the predictive model. Then adopting the optimization and feedback compensation, one optimal control series can be determined by optimal calculating for one performance index. In essence, prediction control take the cost of using part optimizing instead of global optimizing, take account of producing reality by feedback and optimizing. So the control effect of prediction control is between optimizing control and PID control (Phillip D. Schnelle, 1997; Wang, 2002; Endra Joeliant, 2011).

4.1 Establish the Predictive Model of Rough Tracking System

The predictive model of rough tracking system is (Chen Qiao, 2009)

$$
Y_{m}(k+1)=G \Delta U(k)+G^{\prime} U(k-1)
$$

If consider the influence of the moment of force fluctuation, the predictive model of rough tracking system is

$$
Y_{m}(k+1)=(G+\Delta G) \Delta U(k)+\left(G^{\prime}+\Delta G^{\prime}\right) U(k-1)
$$

In the above two expressions:

$Y_{m}(k)$ is the output matrix of predictive mode of future $\mathrm{P}$ sample periods, $\mathrm{G}$ is dynamic matrix, $\mathrm{U}(\mathrm{K})$ is controlling amount matrix, $\Delta \mathrm{U}(\mathrm{K})$ is the increment of controlling amount matrix, $\Delta \mathrm{G}$ is system parameter uncertainty. $\mathrm{N}$ is model length, $\mathrm{M}$ is control time domain, $\mathrm{P}$ is optimization time domain, $g_{i}(i=1,2, \cdots N)$ is the amplitude of impulse response in sampling moment.

$$
\begin{gathered}
Y_{m}(k+1)=\left[y_{m}(k+1 / k), y_{m}(k+2 / k), \cdots y_{m}(k+p / k)\right]^{T} \\
G=\left[\begin{array}{cccc}
g_{1} & 0 & \cdots & 0 \\
g_{2} & g_{1} & \cdots & \vdots \\
\vdots & \vdots & \vdots & 0 \\
g_{p} & g_{p-1} & \cdots & g_{p-m+1}
\end{array}\right]_{P \times M}
\end{gathered}
$$




$$
\begin{aligned}
& G^{\prime}=\left[\begin{array}{ccccc}
g_{n}-g_{n-1} & g_{n-1}-g_{n-2} & \cdots & g_{3}-g_{2} & g_{2} \\
0 & g_{n}-g_{n-1} & \cdots & g_{4}-g_{3} & g_{3} \\
\vdots & \vdots & \ddots & \cdots & \cdots \\
0 & 0 & g_{n}-g_{n-1} & \cdots & g_{p+1}
\end{array}\right]_{P \times N} \\
& \Delta G=\left[\begin{array}{cccc}
\Delta g_{1} & 0 & \cdots & 0 \\
\Delta g_{2} & \Delta g_{1} & \cdots & \vdots \\
\vdots & \vdots & \ddots & 0 \\
\Delta g_{p} & \Delta g_{p-1} & \cdots & \Delta g_{p-m+1}
\end{array}\right]_{P \times M} \\
& \Delta G^{\prime}=\left[\begin{array}{ccccc}
\Delta g_{n}-\Delta g_{n-1} & \Delta g_{n-1}-\Delta g_{n-2} & \ldots & \Delta g_{3}-\Delta g_{2} & \Delta g_{2} \\
0 & \Delta g_{n}-\Delta g_{n-1} & \ldots & \Delta g_{4}-\Delta g_{3} & \Delta g_{3} \\
\vdots & \vdots & \ddots & \ldots & \ldots \\
0 & 0 & \Delta g_{n}-\Delta g_{n-1} & \ldots & \Delta g_{p+1}
\end{array}\right]_{P \times N}
\end{aligned}
$$

\subsection{Feedback Compensation}

Because the system has various disturbances, so the predictive should be corrected. If $y(k)$ is the real output of the rough tracking system, $\mathrm{e}(\mathrm{k})$ is the error of predictive model, then

$$
e(k)=y(k)-y_{m}(k)
$$

The predictive output can be corrected by error weight matrix $h$, and then the predictive output can be expressed as

$$
Y_{p}(k+1)=Y_{m}(k+1)+h e(k)=(G+\Delta G) \Delta U(k)+\left(G^{\prime}+\Delta G^{\prime}\right) U(k-1)+h e(k)
$$

In the above expression:

$$
\begin{aligned}
& Y_{p}(k+1)=\left[y_{p}(k+1), y_{p}(k+2), \cdots y_{p}(k+p)\right]^{T} \\
& h=\left\lfloor\begin{array}{llll}
h_{1}(k) & h_{2}(k) & \cdots & h_{p}(k)
\end{array}\right\rfloor
\end{aligned}
$$

\subsection{Optimization Design}

By comparing the real output of the rough tracking system and the predictive output after feedback compensation, the quadratic form optimum performance index of predictive error can be expressed as

$$
J_{P}(k)=\left[Y_{m}(k+1)-Y_{r}(k+1)\right]^{T} Q\left[Y_{m}(k+1)-Y_{r}(k+1)\right]+\Delta U^{T}(k) R \Delta U(k)
$$

In the above expression, $\mathrm{Q}$ is predictive output error weight matrix, $\mathrm{R}$ is controlling amount weight matrix.

\subsection{Simulation of the APT Rough Tracing Subsystem Based on Predictive Control}

Let the sample period $T_{s}$ is $0.02 \mathrm{~S}, \mathrm{P}=5, \mathrm{M}=5, \mathrm{~N}=20, \mathrm{Q}$ and $\mathrm{R}$ is unit matrix. $h=\left[\begin{array}{llll}1 & 0.2 & \cdots & 0.2\end{array}\right]_{N \times 1}^{T}$, $\Delta G=0.1 G$.

According to the transfer function of the uncompensated rough tracking system, the dynamic matrix can be determined as 


$$
\begin{aligned}
G & =\left[\begin{array}{ccccc}
192.9542 & 0 & 0 & 0 & 0 \\
392.6568 & 192.9542 & 0 & 0 & 0 \\
592.3239 & 392.6568 & 192.9542 & 0 & 0 \\
791.9912 & 592.3239 & 392.6568 & 192.9542 & 0 \\
991.6584 & 791.9912 & 592.3239 & 392.6568 & 192.9542
\end{array}\right] \\
\Delta G & =\left[\begin{array}{ccccc}
19.29542 & 0 & 0 & 0 & 0 \\
39.26568 & 19.29542 & 0 & 0 & 0 \\
59.23239 & 39.26568 & 19.29542 & 0 & 0 \\
79.19912 & 59.23239 & 39.26568 & 19.29542 & 0 \\
99.16584 & 79.19912 & 59.23239 & 39.26568 & 19.29542
\end{array}\right]
\end{aligned}
$$

We can obtain the predictive error is

$$
e=\left[\begin{array}{r}
0.0000 \\
-0.5664 \\
-1.1245 \\
-1.2597 \\
-1.1200
\end{array}\right]
$$

The step response of the rough tracking system based on predictive control is shown in Figure 5, and the system overshoot of the system is $\sigma_{P}=14 \%$, the settling time is $0.9 \mathrm{~S}$, and the steady-state error of the system is 1.6877e-007. The closed loop frequency characteristic of the position loop is seen in Figure 6, and the bandwidth of the position loop is $46.8 \mathrm{rad} / \mathrm{s}(7.45 \mathrm{~Hz})$, which could satisfy the bandwidth requirement of the APT system for the rough tracing subsystem.

In order discuss the influence of system parameter uncertainty, separately let $\Delta \mathrm{G}=0.1 \mathrm{G}, \Delta \mathrm{G}=0.3 \mathrm{G}, \Delta$ $\mathrm{G}=0.5 \mathrm{G}$, the step response of system is shown in Figure 7, from which we can see that the larger the $\Delta \mathrm{G}$ is, the longer the settling time, the larger the overshoot is, the poorer the system performance is.

\section{Conclusions}

In the design of the algorithm of the rough tracking system, in order to overcome the deficiency that the traditional PID and enhance the tracking precision of the rough tracking system, the dynamic matrix control algorithm is used into the rough tracing system. Simulation result shows that the rough tracing system has good dynamic response characteristics. It can satisfy the requirements of the APT system for the rough tracing subsystem.

\section{References}

Chen Qiao. (2009). Research on a class of uncertain DMC method in blast furnace. Hangzhou Dianzi University, 8-19.

Endra Joeliant, Edwina Maryami Sumarjono, Agus Budiyono, \& Dini Retnaning Penggalih. (2011). Model predictive control for autonomous unmanned helicopters. Aircraft Engineering and Aerospace Technology, 83, 375-387. http://dx.doi.org/10.1108/00022661111173252

$\mathrm{Hu}$, Qinggong \& Wang, Kejia. (2005). Design of atmospheric laser communication APT system and accurate tracking system simulation. Application science and technology, 32(12), 7-9.

Liu, Ximin, Sun, Liren \& Sun, Jianfeng. (2006). Bandwidth Design of Composite Axis System in Satellite Laser Communication. Acta Optica Sinica, 26(1), 103-103.

Phillip D. Schnelle, \& David L. Rollins. (1997). Industrial model predictive control technology as applied to continuous polymerization processes. Consultant Process Control, 36, 281-292.

Shinhak Lee, James W. Alexander, \& Muthu Jeganathan. (2000). Pointing and Tracking Subsystem Design for 
Optical Communications Link between the International Space Station and Ground. SPIE, 150-157.

Tong Luo, \& Yu Hu. (2002). Vibration suppression techniques for optical inter-satellite communications. IEEE, 47(8), 356-682.

Toyoda Masahiro. (2006). Acquisition and tracking control of satellite-borne laser communication systems and simulation of downlink fluctuations. Optical Engineering (S0091-3286), 45(3), 4-12.

Tzung-Hsien Ho, Stuart D. Milner, \& Christopher C. Davis. (2005). Fully optical real-time pointing, acquisition and tracking system for free space optical link. Proc.SPIE, 5712, 81-92. http://dx.doi.org/10.1117/12.590982

Wang Chang-ming, \& LEI Rong-xiao. (2002). Application of multivariables predictive control technology in atmosphere and vacuum distillation unit. Petrochemical Technology \& Application, 5.

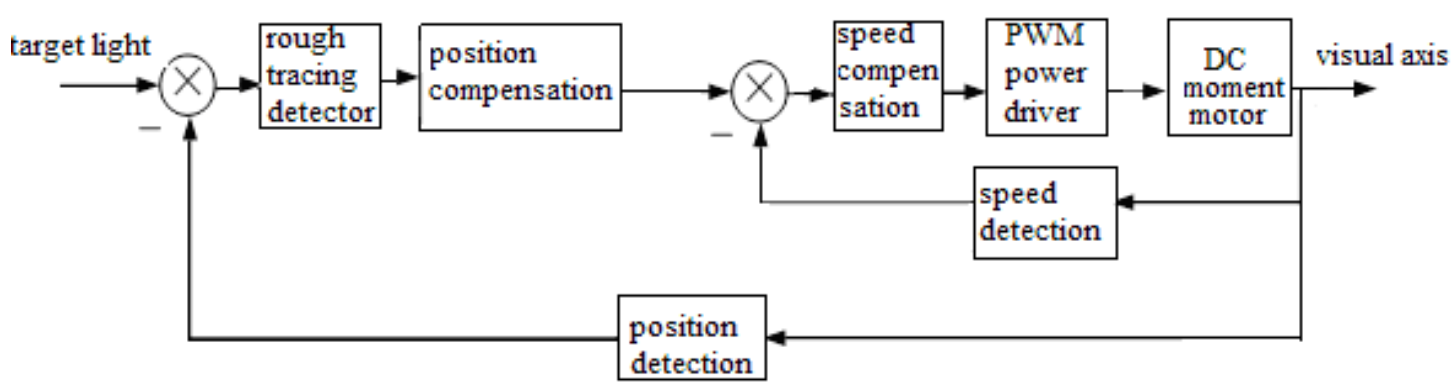

Figure 1. Block Diagram of APT System

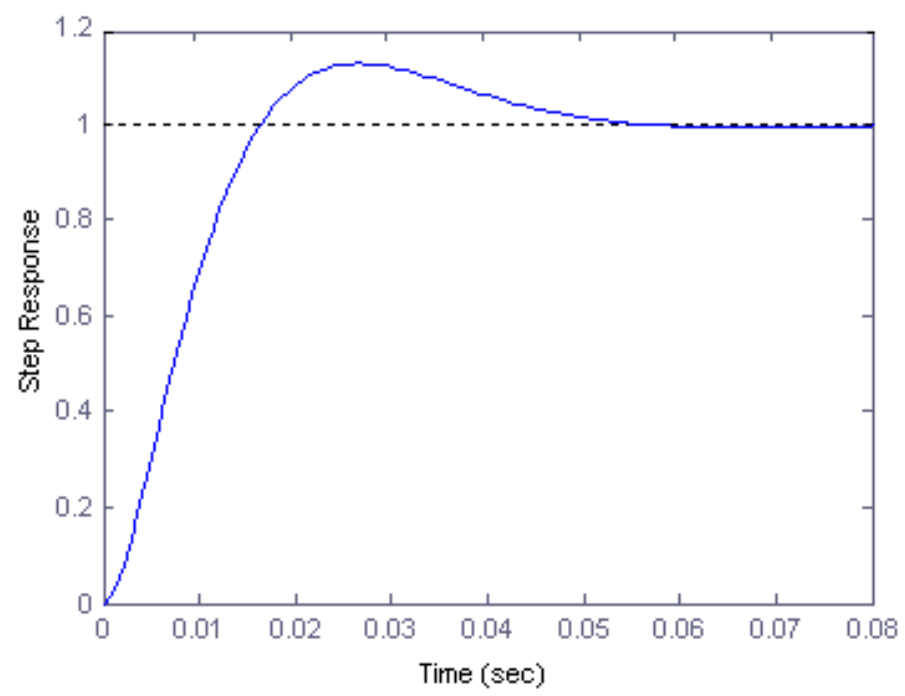

Figure 2. Step Response of Compensated Speed Loop 
Bode Diagram
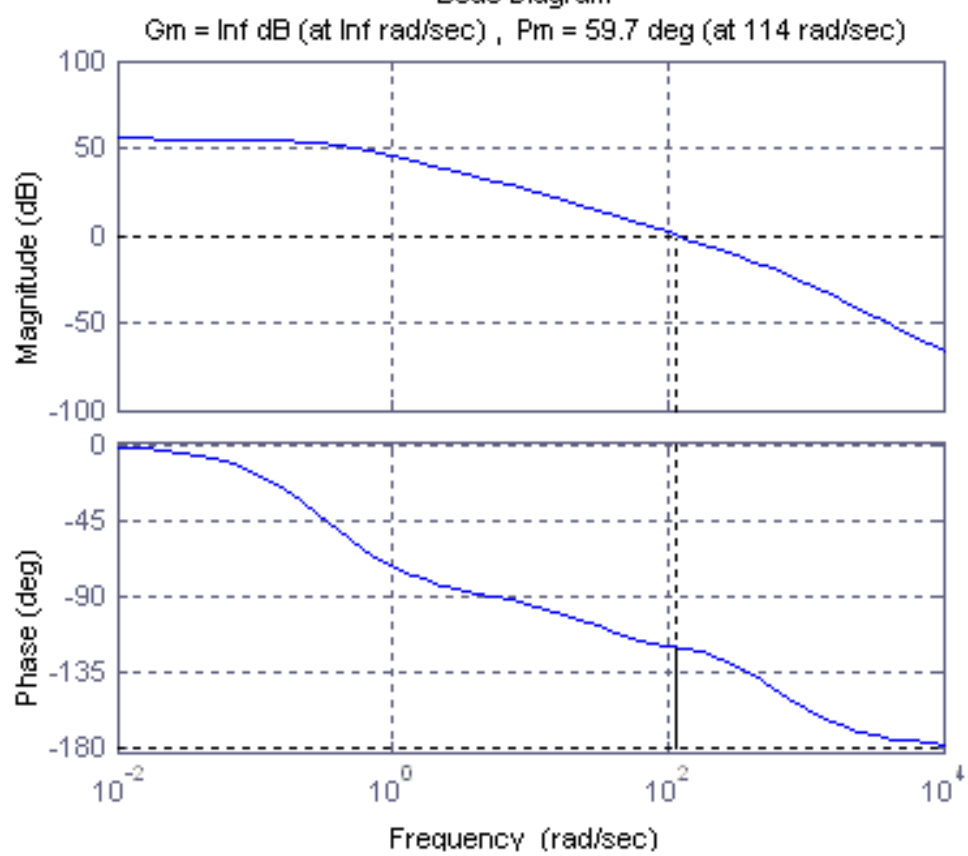

Figure 3. Open Loop Frequency Characteristic of Compensated Speed Loop

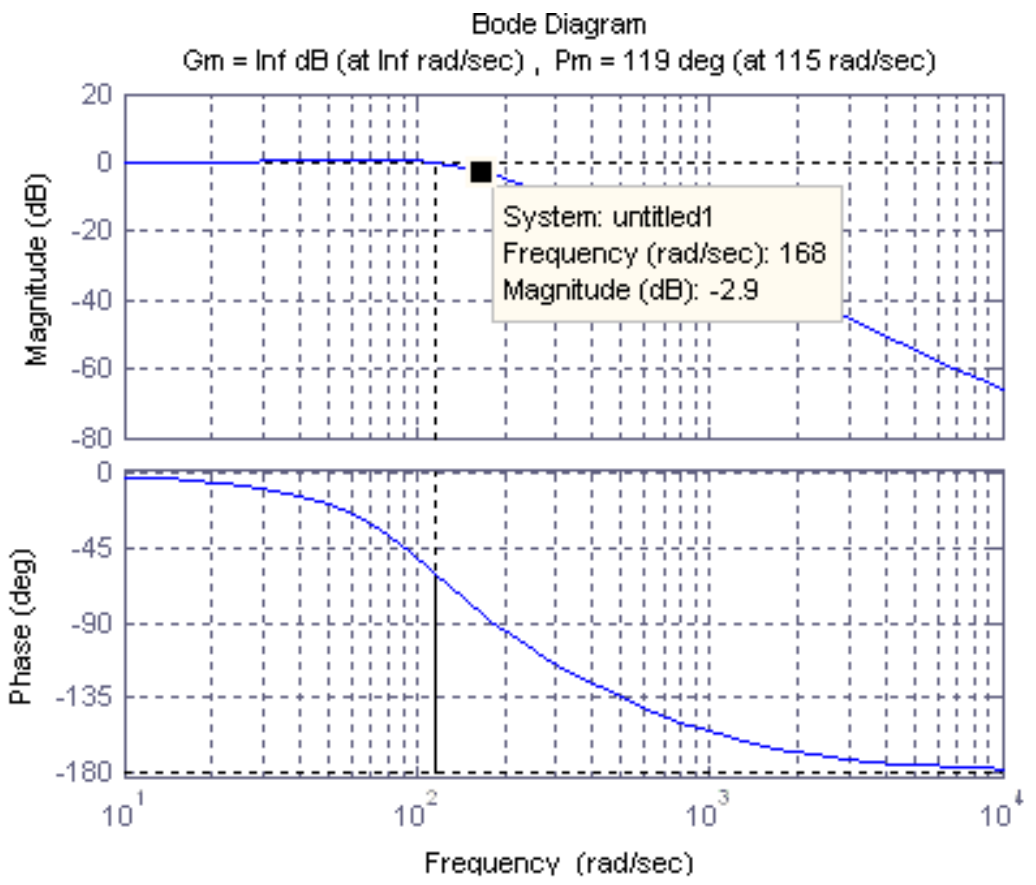

Figure 4. Closed Loop Frequency Characteristic of the Compensated speed Loop 


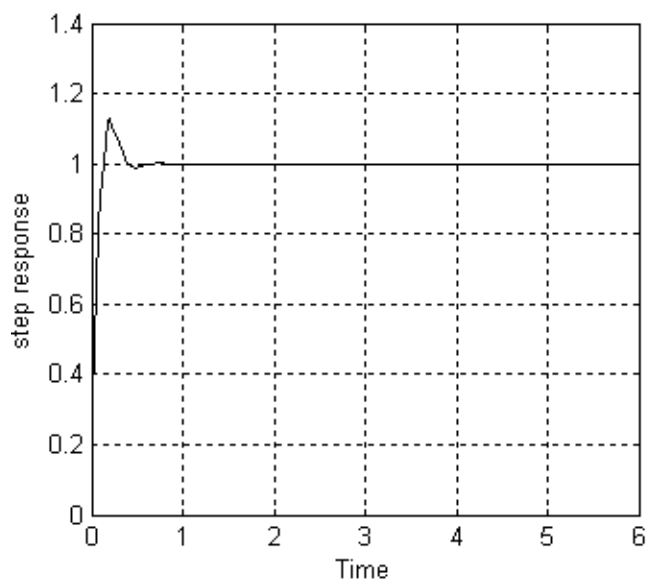

Figure 5. Step Response of Position Loop Based on Predictive Control

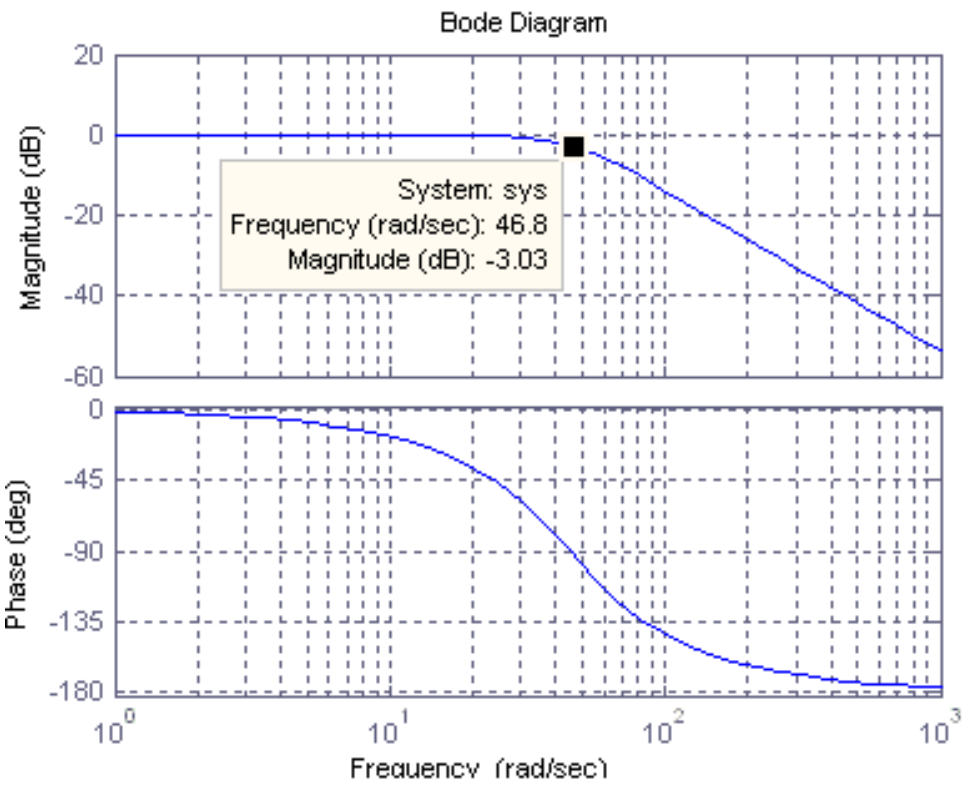

Figure 6. Closed Loop Frequency Characteristic of the Compensated Position Loop

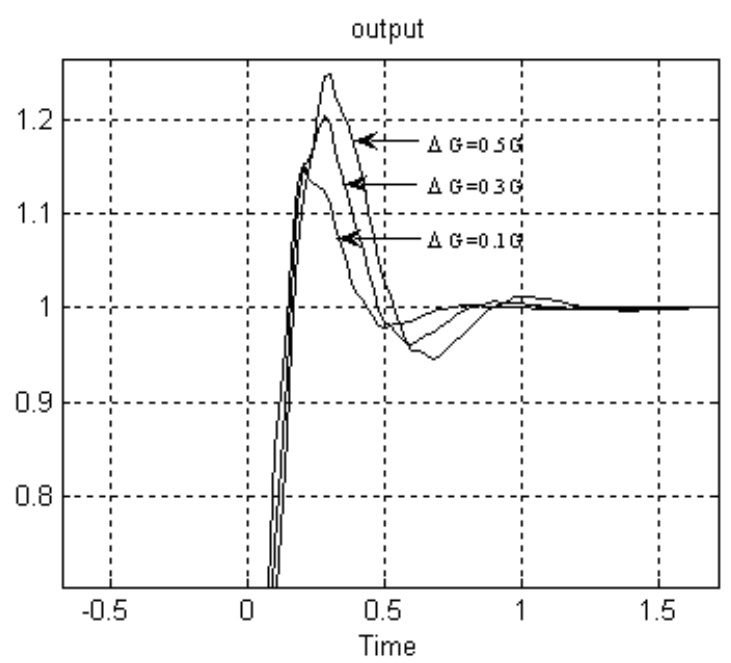

Figure 7. Step Response of Position Loop for Different $\Delta G$ 